Bull. Mater. Sci., Vol. 22, No. 3, May 1999, pp. 585-592. (C) Indian Academy of Sciences.

\title{
Microstructures in Al-rich $\gamma$-TiAl strained in the domain of temperature of flow stress anomalies
}

\author{
FABIENNE GRÉGORI and PATRICK VEYSSIÈRE* \\ LEM, CNRS-ONERA, BP72, 92322-Châtillon Cedex, France
}

\begin{abstract}
The plastic behaviour of single-phase $\boldsymbol{\gamma}$-TiAl poses a certain number of fundamental problems as to the origin of the flow stress anomaly and to how this is related to dislocation properties. Understanding these questions is of significant interest in the investigations of flow stress anomalies in intermetallics and in other materials. Also, it may reveal useful in the study of the $\gamma$-based lamellar alloys. The present contribution accounts for an investigation of mechanical properties and microstructural organization conducted in Al-rich $\gamma$-TiAl single crystals oriented to activate single slip. Three separate topics are addressed.

(I) $1 / 2\langle 110]$ ordinary dislocations after deformation between room temperature and $800^{\circ} \mathrm{C}$. The magnitude of the cusping appears to depend on the chronology of dislocation immobilization. The distribution of pinning points is analysed and the temperature dependence of their density confronted to that of the flow stress.

(II) The core structure of (011] dislocations. After deformation at room temperature, [011] dislocations are dissociated into two partials separated by a stacking fault. The role of temperature and possible transitions of the dissociation are addressed.

(III) The observation and analysis of background striations which cover the thin foils entirely. The striations are shown to be a deformation by-product resulting from the passage of perfect dislocations of the $L 1_{0}$ structure, and from the interaction of these with a short-range ordered ( $\mathrm{SRO}) \mathrm{Ti}_{3} \mathrm{Al}_{5}$ phase. Striations can serve to discuss the absence of slip localization and the properties of cross-slip.
\end{abstract}

Keywords. $\gamma$-TiAl; single crystal; slip systems; flow stress; dislocations; deformation microstructure.

\section{Introduction}

The present work is part of an extensive study of the plastic properties of TiAl-based alloys. Of interest for potential aerospace applications, the lamellar two-phase $\gamma / \alpha 2$ alloy is located on the Ti-rich side of the phase diagram. These alloys, the object of intensive investigations worldwide, exhibit plastic properties which are largely determined by the nature of the various interfaces between the $\gamma$-variants and the $\alpha 2$ lamellae. In view of the limited thickness of the lamellae relative to the order of magnitude of the mean free-flight distances of dislocations, lamellar TiAl alloys offer but limited opportunity to elucidate the properties of individual dislocations, hence to discriminate between an interface- and a core-controlled behaviour. Determining whether the latter may or may not play a role during deformation, together with the extent of this role, thus requires the support of reference observations conducted in interface-free samples. It is in this spirit that we have undertaken dedicated studies of the local properties of dislocations and of the relations of these properties with the macroscopic deformation behaviour. The alloy compositions investigated here were chosen within the Al-rich side of the Ti-Al phase diagram where the $\gamma$-TiAl phase (ordered $\mathrm{LL}_{0}$ structure) is in

*Author for correspondence equilibrium. A further interest for Al-rich TiAl alloys relies in the variety of atypical mechanical properties thus offered to investigations and the possibility to extend our insight on the various causes of a flow stress anomaly in alloys. Preliminary studies have indicated that, when proceeding by ordinary dislocations, the flow stress peak coincides with a significant cross-slip activity, suggesting in turn that the former controls the plastic flow through a process which is intrinsic to the core of ordinary dislocations. There are some differences though between groups as to the details of the cross-slip anchoring process (Viguier et al 1995; Sriram et al 1997). Worthy of mention is the alternative point of view developed by several groups whereby the cusping is controlled by (invisible) particles or clusters interspersed in the $\gamma$ matrix and, again, point of views may differ as to the nature of the particles (Messerchmidt et al 1995; Nakano et al 1996; Wiezorek and Fraser 1998; Zghal et al 1998). Clearly in this case, the strengthening would be largely extrinsic to the dislocation cores.

Inherent to the $\mathrm{Ll}_{0}$ structure is the multiplicity of potential slip directions $(b=1 / 2\langle 110],\langle 101], 1 / 2\langle 112]$, and $\langle 001]$, the latter being activated at elevated temperatures only (Hug 1988, figure 1) and the resulting difficulty in isolating the properties of each of the three possible families of perfect dislocations in randomly oriented grains. Single crystals of Al-rich TiAl are on 
the other hand, difficult to grow requiring specific equipment that make monocrystalline single phase rods a rare item. As a consequence, the number of dedicated studies of dislocation microstructures tailored under wellcontrolled thermomechanical conditions are relatively limited (Stucke et al 1995; Inui et al 1997; Jiao et al 1998). It is by far less than, for example, what was revealed necessary in the past to elucidate the deformation properties, related microstructures and dislocation properties of $\mathrm{L}_{2}$ alloys (Nabarro and Duesbery 1996). The present investigation is aimed at contributing to the collective endeavour undertaken on TiAl alloys. We report here on results which we have obtained mostly by transmission electron microscopy, both on $1 / 2\langle 110]$ ordinary and $\langle 011]$ (super)-dislocations.

\section{Experimental}

We report here our studies on poly- and monocrystalline samples with a composition located in the vicinity of 54.5 at\% Al (say, $54.5 \pm 0.5$ ). This is approximately the minimum aluminium content above which single crystals could be successfully and more or less reproducibly grown so far. Parallelipedic samples $(3 \times 3 \times 8 \mathrm{~mm})$ were deformed in compression between room temperature and $800^{\circ} \mathrm{C}$ so as to cover the domain of temperature where the flow stress first exhibits a plateau-like behaviour, then peaks $\left(600-750^{\circ} \mathrm{C}\right.$, depending on orientation and composition) and subsequently decreases (Kawabata et al 1985; Inui et al 1997). The deformation was achieved under a constant strain rate of $5 \times 10^{-5} \mathrm{~s}^{-1}$.

In view of the limited volume of single crystal available (4 samples per orientation, 2 orientations), our tests had to be limited to a few deformation temperatures which we have selected for their location in the temperature dependence of the flow stress (i.e. room temperature, $400^{\circ} \mathrm{C}, 600^{\circ} \mathrm{C}$ and $800^{\circ} \mathrm{C}$ ). We have excluded the peak temperature by itself as well as the domain of transition between the plateau and the ascending part of the flow stress, for these are suspected to involve more than one deformation mechanism (at the peak for instance, one expects a mixture of the hardening and softening processes which, in TiAl, appear to be controlled by mechanisms as different as glide and climb, respectively). By contrast, the investigation that we have carried out on polycrystals is more extensive. It has however provided but modest unambiguous information on dislocation behaviour because of the multiplicity of activated slip systems, of the difficulty in preparing thin foils that are conveniently oriented with respect to the operating slip plane(s) and of the uncertainties on the Schmid factors that correspond to the dislocations studied.

'Itself known to activate slip of ordinary dislocations while the (011] \{111\} dominates everywhere else (Inui et al 1997).
As to the investigation on single crystals, we have chosen two load orientations, [1 53 ] and [ 1216 2 6 . The criterion for this choice is that the load axis be sufficiently close to the [021] orientation ${ }^{\dagger}$, for the $\langle 110]\{111\}$ slip system to be favoured in both cases. The two orientations have nevertheless significantly distinct Schmid factors on planes other than the primary octahedral plane, and this was designed in order to influence cross-slip differently (table 1) hence, to better evidence and quantify the role of cross-slip in the locking process.

From each deformation sample, two sets of thin foils have been sliced parallel either to the expected operating octahedral slip plane or to the octahedral cross-slip plane. These foils were studied by transmission electron microscopy under weak-beam conditions at $200 \mathrm{kV}$.

\section{Results}

Whereas the four samples deformed along [1 53 3] between room temperature and $800^{\circ} \mathrm{C}$ all responded as expected by slip on $\langle 110]\{111\}$, the tests conducted along the [1 216 6] orientation revealed at first sight quite disappointing results for most of the foils contained profuse [011] dislocations, while the wholesale appearance of the microstructure varied quite dramatically from one test temperature to the next. As shown below, this nevertheless enabled us to conduct two independent studies on each slip system and to address a rather reasonable set of dislocation properties which we summarize below.

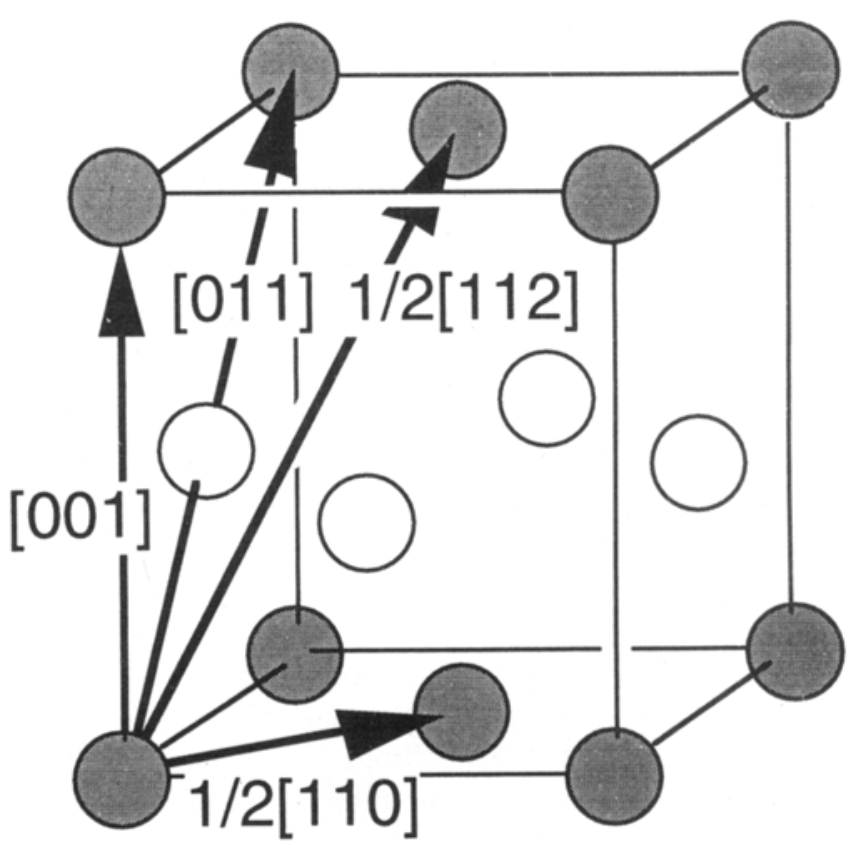

Figure 1. The unit cell of TiAl and the representation of all the unit translations which a dislocation may adopt as a Burgers vector. 
Table 1. A tabulation of the most significant Schmid factors for the two load orientations investigated.

\begin{tabular}{|c|c|c|c|c|c|c|c|}
\hline & {$[110](\overline{11} 1)$} & {$[110](1 \overline{1} 1)$} & {$[110](001)$} & {$[110](\overline{1} 10)$} & {$[1 \overline{10}](111)$} & {$[1 \overline{1} 0](11 \overline{1})$} & {$[\overline{10} 0](001)$} \\
\hline$\left[\begin{array}{lll}1 & 5 & 3\end{array}\right]$ & 0.490 & -0.061 & 0.367 & -0.339 & -0.418 & -0.132 & -0.245 \\
\hline \multirow[t]{2}{*}[\begin{array}{lll}{1}&{21}&{6}\end{array}]{} & 0.488 & -0.256 & 0.199 & -0.459 & -0.478 & -0.267 & $-0 \cdot 180$ \\
\hline & {$[\overline{1} 01](111)$} & {$[\overline{10} 01](1 \overline{1} 1)$} & {$[\overline{1} 01](010)$} & {$[101](111)$} & {$[101](\overline{1} 11)$} & {$[101](010)$} & {$[011](11 \overline{1})$} \\
\hline$\left[\begin{array}{lll}1 & 5 & 3\end{array}\right]$ & $0 \cdot 220$ & -0.021 & $0 \cdot 210$ & 0.333 & $0 \cdot 135$ & 0.408 & $0 \cdot 266$ \\
\hline$\left[\begin{array}{lll}1 & 2 & 1\end{array}\right]$ & $0 \cdot 124$ & -0.060 & $0 \cdot 161$ & $0 \cdot 159$ & 0.096 & 0.222 & 0.360 \\
\hline
\end{tabular}

\subsection{The $\langle 110]\{111\}$ slip system}

With regard to the locking of ordinary dislocations by double cross-slip, one question of interest is to ascertain the crystallographic nature of the cross-slip plane. Both $\{111\}$ and $\{100\}$ have been reported so far (Sriram et al 1997). In the course of our experiments on poly- and monocrystals, we have actually encountered quite a variety of cross-slip planes including the above two planes as well as some instances of $\{110\}$. It appears that, given a Schmid factor on the operating slip system and a deformation temperature, the cross-slip plane is not unique. We have found that cross slip either on $\{001\}$ or on $\{110\}$ may occur in places adjacent to regions dominated by primary octahedral slip, thus questioning the role of the applied stress in the cross-slip process.

The most striking though well known microstructural characteristics of $\langle 110\}\{111\}$ slip are (i) the overwhelmingly cusped aspect of ordinary dislocations and (ii) the predominant alignment of these with the screw direction. This behaviour of the ordinaries is encountered to varied extents at every temperature tested below the peak, but it is most prominent in the $400^{\circ} \mathrm{C}-600^{\circ} \mathrm{C}$ range (figure 2). After deformation at room temperature, the proportion of screw segments is decreased with respect to what is encountered after deformation at higher temperatures in the flow-stress plateau. Above the peak temperature, the microstructure is dramatically different consisting essentially loop-forming mixed dislocations. At this latter temperature $\left(800^{\circ} \mathrm{C}\right)$, there is ample evidence for climb, as already reported by several groups. Confirming the observations made by a number of groups and despite many dedicated attempts, we have been unable to find any evidence of a possible dissociation into Shockley partials, which the symmetry of the L1, structure allows in principle.

With regard to ordinary dislocations operating at intermediate temperatures, what our observations have brought about is that the screw dislocations are arrested in bundles as opposed to being randomly interspersed in the foil. One example of such arrangements observed in foils parallel to the slip plane is shown in figure 3 . In many examples, though not all, there is indication that the amplitude of the cusping decreases from the leading to the trailing dislocation. Observations conducted in foils cut at about $70^{\circ}$ from the primary slip planes have revealed that the piling up is by no means coplanar. Some stress enhancement is nevertheless expected at the spearhead of the bundle, though certainly not as dramatic as in the case of a coplanar pile up. For such arrangements to be stable, the lattice has to oppose quite a resistance to dislocation motion. Another evidence of the strength of lattice friction against octahedral slip of ordinary dislocations is provided by the cusping by itself, which is certainly not an equilibrium configuration either. The remanence of the bowing out is further illustrated in figure 3 by the array of truncated half-loops whose extremities both emerge at the same free surface and which, in the absence of lattice friction, would have spontaneously self-annihilated under the influence of its attractive image force. Tilting experiments have in addition confirmed the previous findings of Sriram et al (1997) that two adjacent cusps may lie on distinct, parallel planes. In addition to the primary octahedral plane, we have found that such configurations could be as well encountered on $\{110\}$ planes. In both cases, there is a jog at the interconnection between the two adjacent bowed out segments, the jog length embodies the trajectory of the screw segment in the cross-slip plane.

\subsection{The $\langle 011][111\}$ slip system}

As mentioned above, deformation along [ [ 1216 does not proceed by ordinary dislocations. In fact, our observations of samples deformed at room temperature indicate the simultaneous presence of the three possible Burgers vectors of the $\mathrm{Ll}_{0}$ structure, $1 / 2\langle 110],\langle 011]$ and $1 / 2\langle 112]$, all simultaneously pertaining to the same octahedral slip plane (figure 4), i.e. no other slip planes are activated. By contrast, the microstructure after deformation at $400^{\circ} \mathrm{C}$ and $600^{\circ} \mathrm{C}$ consists of a majority of $\langle 011]$ dislocations under single octahedral slip, with a frequency of ordinaries and $1 / 2\langle 112]$ dislocations that decreases gradually with temperature. 
Regarding the lowest deformation temperatures whose deformation microstructures coincide with a slip activity in multiple directions, what occurs may be either one of the following two scenarios: (i) two slip directions are activated in the same slip plane, interacting in places to form $1 / 2\langle 112$ ] junctions, themselves essentially sessile at this temperature (Hug et al 1988); or (ii) the <011] dislocation is activated but the stress tensor helps for its spontaneous decomposition into an ordinary and a $1 / 2\langle 112]$ dislocation

$$
[011] \rightarrow 1 / 2[\overline{1} 10]+1 / 2[112]
$$

and this is because the stress tensor acts on these dislocation by-products differently. The $1 / 2\langle 112]$ dislocation would thus constitute the trace of the unzipping

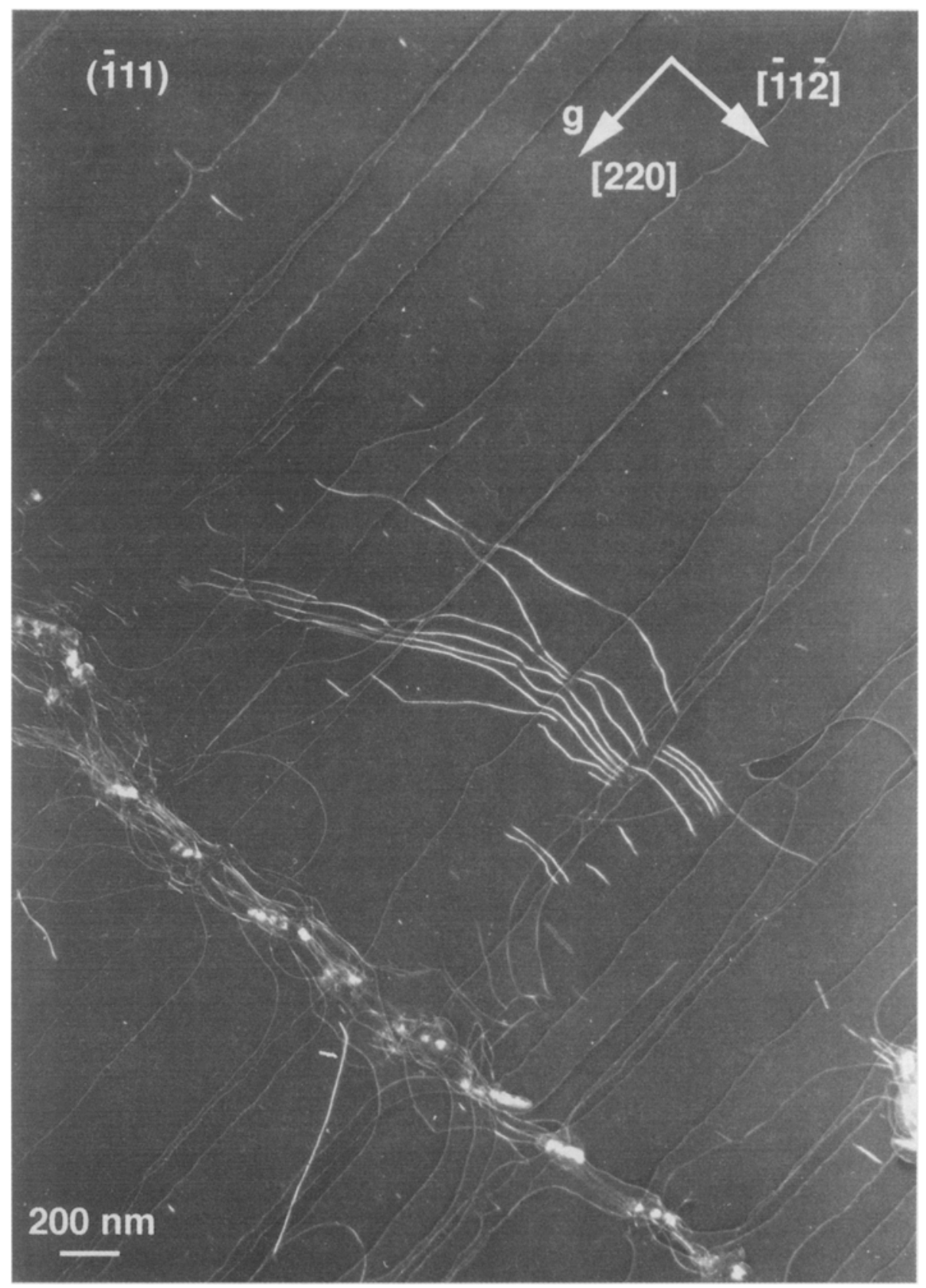

Figure 2. The microstructure after deformation under [110](111) single slip at $400^{\circ} \mathrm{C}$. Dislocations show a marked preference for the screw orientation. The fact that the mixed parts are much more mobile than the screws is illustrated by the dislocation entanglement built up against the wall of precipitates $\left(\mathrm{Ti}_{2} \mathrm{AIX}, \mathrm{X}=\mathrm{C}, \mathrm{N}, \mathrm{O}\right)$. Clearly, the applied stress was not enough to help dislocations overcome the obstacle array. This caused the accumulation of every mobile segments which, otherwise, would have disappeared at the closest sinks. The cusped aspect of the screws should be noted (see also figure 3 ). 
of ordinaries off the moving (011] dislocations. The latter explanation is however not at first sight consistent with predictions based on an analysis of the various Schmid factors since both by-products of the decomposition reaction exert a force in the same sense. There remains however the possibility that the forces on each decomposition by-product together with the mobility of the two of them be significantly different so that there is a finite mechanical driving force for an ordinary dislocation to escape dynamically from the mobile <011] dislocation.

By contrast with the properties of ordinary dislocation, [011] dislocations show clear evidence of a dissociation. Whether the dissociation is two- or three-fold, together with the question of the dissociation habit plane are beyond the scope of the present paper. Instead, what we should like to emphasize here are the unusual properties of the contrast and the difficulties thus introduced in the analysis of the fine structure of $\langle 011]$ dislocations. There are indeed two possible alternatives for the dissociation of a $\langle 011]$ dislocation into two partials. These reactions differ in the nature of the interconnecting planar defect, that is, either a stacking fault or an antiphase boundary. Namely

$$
\begin{aligned}
& {[011] \rightarrow 1 / 6[112]+\mathrm{SF}+1 / 6[\overline{15} 5],} \\
& {[011] \rightarrow 1 / 2[011]+\mathrm{APB}+1 / 2[011] .}
\end{aligned}
$$

For the sake of simplicity, the various possible subdissociations to form superlattice stacking faults are ignored. We have observed that (i) given the direction of the diffracting vector $\mathbf{g}$, both partials can be in contrast, exhibiting about the same intensity but that, (ii) upon the reversal of $\mathbf{g}$ (or equivalently, of the deviation from Bragg conditions $s_{\mathrm{g}}$ ), the same configuration happens to exhibit a single contrast. In the former case, the contrast is very much like that of a $\langle 011]$ dislocation dissociated into two like partials (reaction 3 ), while in the latter one may conclude that the dissociation is of the SF-couple type (reaction 2), with the Shockley partial set out of contrast. With the help of image simulations, we have determined that the operating dissociation mode is reaction (2) after deformation at room temperature and $400^{\circ} \mathrm{C}$, while it can be a mixture of both reactions (2) and (3) at $600^{\circ} \mathrm{C}$.

We have in addition observed that the locking into Kear-Wilsdorf-like configurations occurs at temperature as low as ambient temperature. Another striking feature of the deformation microstructure under [011] slip, is indeed the locking of screw segments of which ample evidence is encountered within the $400^{\circ} \mathrm{C}-600^{\circ} \mathrm{C}$ range. After room temperature, these locks are much less frequent but they are definitely present. There is indication that their core is three-dimensional which, because of the narrowness of the dissociations, makes it almost impossible to elucidate the actual dissociation mode under

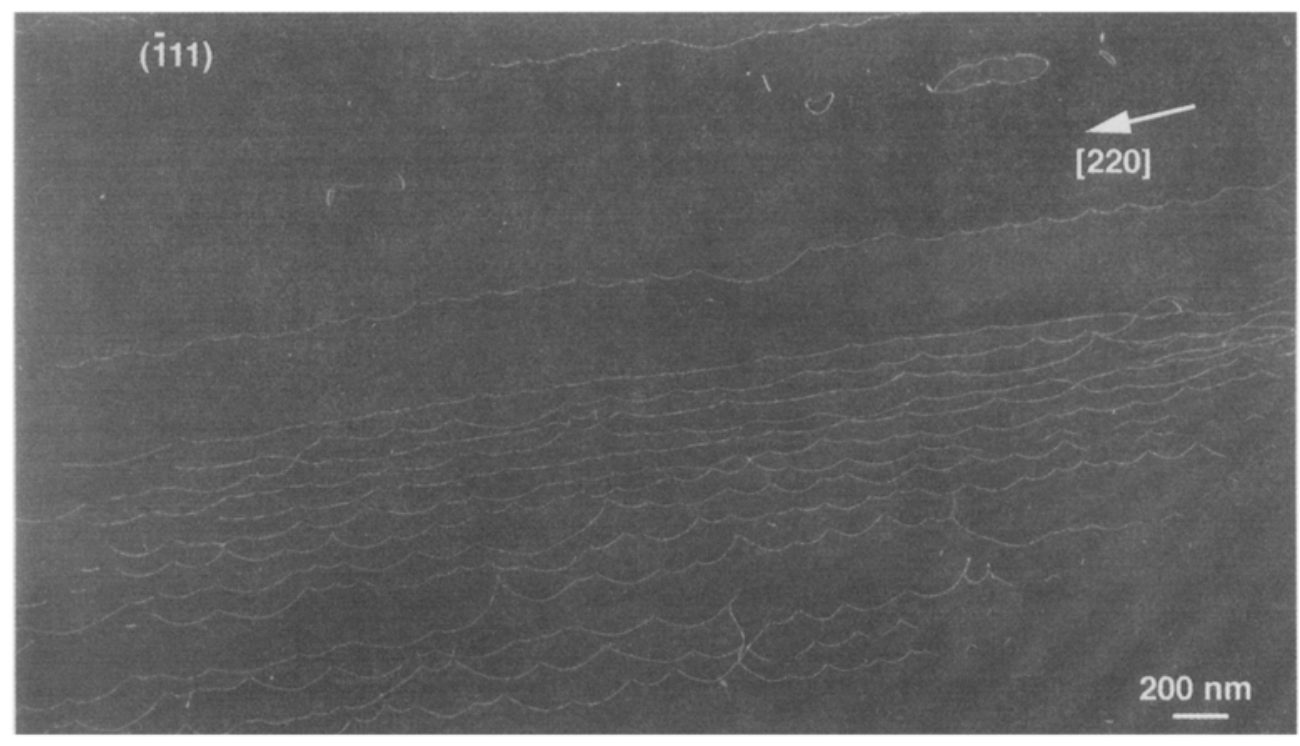

Figure 3. An example of the organization of cusped ordinary screw dislocations in bundles. The magnitude of the cusping changes continuously from one dislocation to the next, with the leading dislocation exhibiting cusps with the largest curvature (the sense of dislocation motion is indicated by the curvature of the cusps). This suggests that the curvature is a function of the order of the dislocation in the bundle, hence of the time it joined the bundle. The presence of alignments of truncated cusps (mostly located at the right-hand side of the figure) should also be noted. 


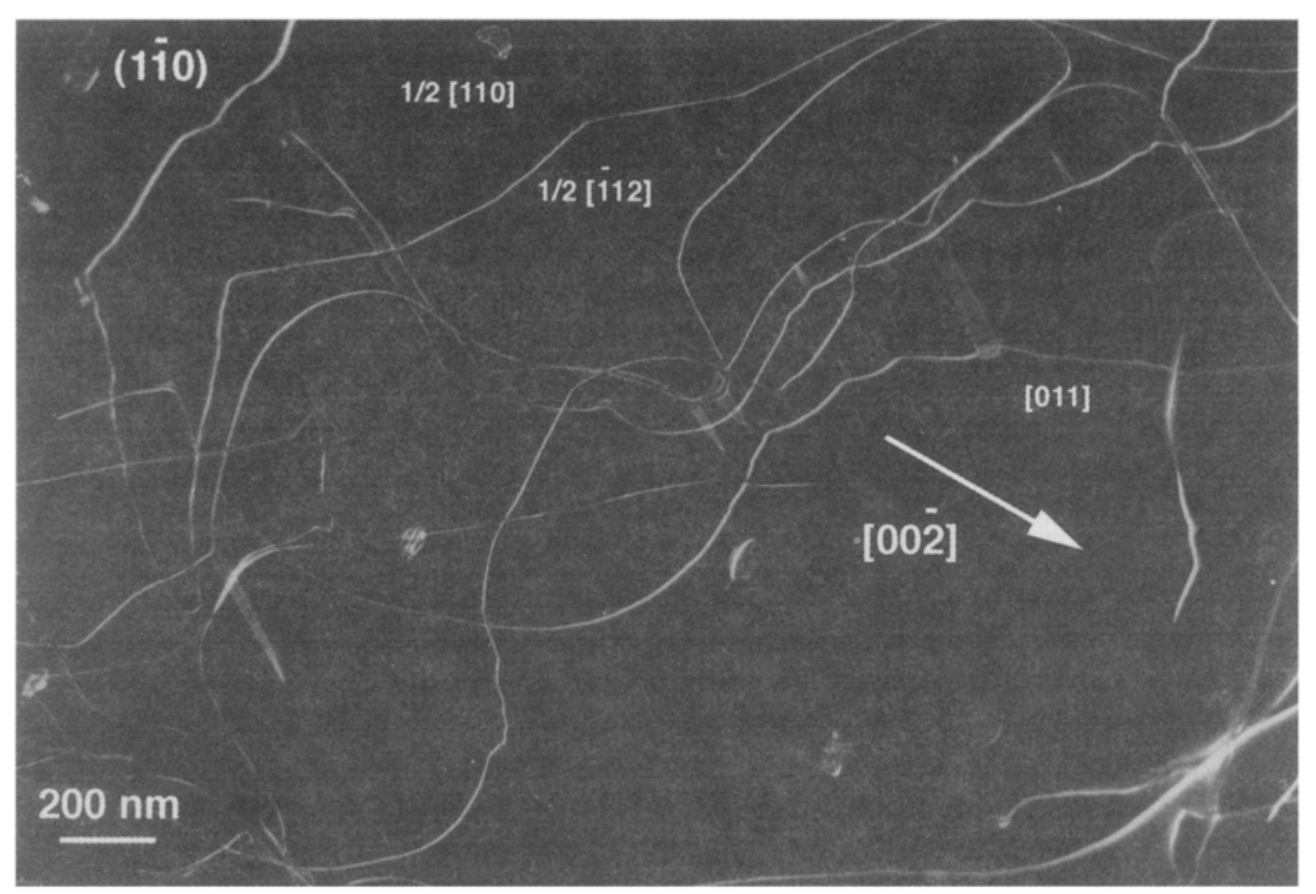

Figure 4. The coexistence of three coplanar slip directions in TiAl deformed at room temperature. Under the 002 reflection the $1 / 2[110]$ are out of contrast, some members of this family show residual contrast in places.

weak-beam conditions. We have nevertheless found that the probable origin of the numerous SF dipoles, known to constitute the signature of 4011$]$ slip, is the result of some pinning at the interconnection between the tip of the screw lock and the adjacent curved mixed segment.

\subsection{Striations}

Above say 54 at\% Al, TiAl is in fact not single phased as indicated by the presence of extra spots and/or of diffuse scattering, which Miida et al (1982), followed by several other workers (Loiseau 1985; Hug 1988; Inui et al 1997, 1998; Nakano et al 1998, 1999), have identified as arising from the $\mathrm{Ti}_{3} \mathrm{Al}_{5}$ phase (Al atoms sit at antisite positions within the $\mathrm{Ti}$ basal planes and assume). In the range of composition of interest here (e.g. $54.5 \pm 0.5$ at $\%$ ), the diffraction patterns of Al-rich TiAl indicate short-range order only. Although this cannot be ignored, it is usually not addressed as a factor capable of influencing dislocation mobility, but by two groups (Nakano et al 1996, 1998; Inui et al 1998). We shall return to this question in $\S 4$.

In the course of our investigations of deformation microstructures, we have noticed the presence of faint fringes (figure 5), referred to hereafter as striations, which cover the thin foils uninterruptedly (Grégori and Veyssière 1999). The reasons why this feature had not been reported previously are essentially two-fold: (i) the contrast is intrinsically faint requiring, in addition to

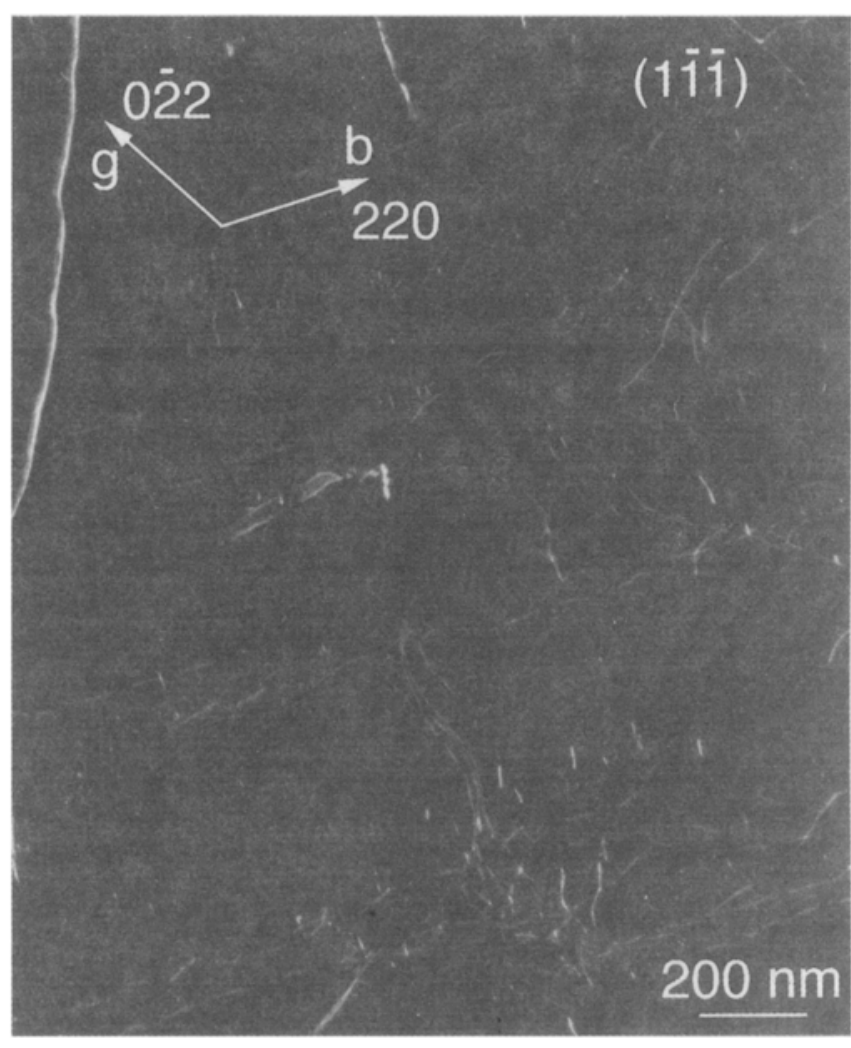

Figure 5. Striations in a foil sliced nearly parallel to the operative (111) plane. The sample was deformed at room temperature along the [153] orientation which activates ordinary dislocations. 
critical imaging conditions, significant contrast enhancement by image processing and (ii) as made clear below, multiple slip operation is detrimental to the visibility of the striations. By appropriate imaging conditions, we have proven that these originate from the bulk of the thin foils (as opposed to being created by some defect located at the free surfaces) and that they are a consequence of plastic deformation, i.e. of the passage of dislocations. Either family of dislocations, e.g. ordinaries and supers, do generate fringes whose aspect is the same irrespective of the active Burgers vector. Striations fade away for straining temperatures beyond $400^{\circ} \mathrm{C}$.

We have determined that the defects at the origin of the contrast are planar in nature and that the contrast is visible under a fundamental reflection, implying in turn an origin other than a fault in the ordered structure (APB). The fact that the striations are uninterrupted over the foils is to out knowledge, totally unprecedented an observation. It implies that the planar defects in question overlap in projection. We have retrieved the overall contrast properties of the striations by image simulations, the main output of which is that the modulus of the displacement vector is of the order of $5 \times 10^{-3}\langle 011]$. Hence, the origin of the striation contrast is most certainly related to slight atomic relaxations.

The interpretation which we have ended up with is as follows (Grégori and Veyssière 1999). Upon gliding in the crystal, dislocations cut through pockets of $\mathrm{Ti}_{3} \mathrm{Al}_{5}$ like short-range order. Although the stacking is preserved (i.e. $\mathrm{Ti}$ and $\mathrm{Al}$ planes alternatively in the [001] direction), the shear introduces a number of first-neighbour violations in the $\mathrm{Ti}$ planes (with respect to the reference $\mathrm{Ti}_{3} \mathrm{Al}_{5}$ structure), hence the source of relaxations. Since no size effects are however expected from differences in atomic radii, the relaxations are thought to originate from the anisotropy of charge densities, known to take place in TiAl (Fu and Yoo 1990; Morinaga et al 1990; Woodward and MacLaren 1996). The fact that striations do not appear in samples deformed above $400^{\circ} \mathrm{C}$ is fully consistent with the above interpretation. This is in fact rather low a temperature for long-range diffusion to take place (dislocation climb is already detected at $800^{\circ} \mathrm{C}$ ), quite enough though for short-range atomic jumps to recover the $\mathrm{Ti}_{3} \mathrm{Al}_{5}$ atomic short-range order.

\section{Discussion and conclusion}

It has very rapidly occurred to the community that the positive dependence of the flow stress-the so-called flow stress anomaly, which is a property commonly encountered in a variety of intermetallic systems-does not stem from a unique physical origin (see Veyssière 1989). In spite of the many analogies found in very distinct crystal structures, such as the predominance of screw dislocations, a number of significantly different microstructural properties imply that one should take the specificity of each system into consideration. Striking under this respect is the case of TiAl. Inui et al (1997) have indeed unambiguously demonstrated that this system exhibits a flow stress anomaly, be the operative slip direction of the $(110]$ or of the $\langle 011]$ type. In the latter case, dislocations show a favoured screw orientation with a fine structure that reminds of the Kear-Wilsdorf locks commonly encountered in $\mathrm{L}_{2}$ alloys $(\$ 3.2)$, hence providing a plausible track to explore in order to interpret the positive temperature dependence of the flow stress. The case of the $1 / 2\langle 110]$ dislocations makes less sense since, despite the presence of long screw segments, there appears no experimental and little theoretical evidence of a locking ability. In particular, ordinary dislocations have no potential for a transformation into a KearWilsdorf-like core structure for they can only dissociate into Shockley partials. Under this particular respect, TiAl is totally similar to a fcc metal with a high stacking fault energy such as $\mathrm{Al}$.

As explained in $\S 3.1$, the specificity of ordinaries in TiAl is their preference for the screw orientation. This effect is commonly explained in terms of charge densities, similar to though not as pronounced as in covalent crystals such as $\mathrm{Si}$. The screws contain in addition a number of pinning points. Consistently, explanations and models that take the pinning into account have been designed (Viguier et al 1995; Messerschmidt et al 1995; Zghal et al 1998; Sriram et al 1997; Wiezorek and Fraser 1998). Some such as that of Louchet and Viguier (1995), based on statistical measurement of pinning point density with temperature, predicts that the flow stress increase stems from the increase of the pinning point density. A rapid analysis of the distribution of cusps over a large number of screw dislocations has allowed us to conclude that the correlation between the distance between pinning points and the flow stress is, to say the least, rather uncertain. In particular, this distance peaks in the region of the flow stress plateau and increases very markedly with temperature at temperatures where, on the other hand, the flow stress peaks. A more refined, quantitative analysis of the geometrical properties of cusps, involving the question of the jog height (Sriram et al 1997), is underway and will be provided in a forthcoming paper.

Nakano et al (1996) have designed a tentative interpretation of the flow stress anomaly, which postulates that SRO constitutes the driving force for locking by cross-slip. Everywhere a volume of SRO is sheared, perfect dislocations of the $\mathrm{L1}_{0}$ structure create firstneighbour violations in their wake, hence a planar defect. This is indeed consistent with the above observations of striations. According to Nakano et al (1996), dislocations would then tend to cross-slip on the planes 
that offer the least back force to their motion. Based on a comparison of broken bonds, these authors have determined that under such conditions there is a finite driving force for cross slip on the cube plane. In the frame of this explanation, it is however unclear as to why in $\mathrm{Ti}-55$ at $\% \mathrm{Al}$, the flow stress would tend to increase at temperatures where SRO is gradually fading out (with respect to say $400^{\circ}-500^{\circ} \mathrm{C}$, in the flow-stress plateau where SRO kinetics is at a maximum).

The flow stress anomaly is in addition far from being exclusive to ordinaries. It is in fact the $\langle 011\}\{111\}$ slip system which predominates for any load orientation but in the vicinity of [021]. It is therefore quite surprising that so little attention has been paid so far to the cause of this anomalous behaviour. Apart from the existence of a locking process, little is known on the mechanisms whereby (011] dislocations move, by-pass the locks and multiply, and how these relate to the overall flow stress increase beyond $600^{\circ} \mathrm{C}$. As shown by the history of investigations on the flow stress anomaly in $\mathrm{L1}_{2}$ alloys (Nabarro and Duesbery 1966), this kind of information is indeed essential in the modelling of the mechanical properties.

In conclusion for this brief overview of the mechanical properties of Al-rich TiAl alloys, the question is almost at its infancy and there is most certainly a long way to go before one gets a clear idea of the processes that take place in this family of alloys. The question of the role of SRO remains to be addressed comprehensively and there is probably much to learn from a comparison between the moderately Al-rich alloy (e.g. about 54.5\%) and alloys with compositions located on both extremes (Al-rich and near stoichiometric). It is particularly important for the understanding of the pinning processes by which the deformation microstructures be satisfactorily characterized in alloys free from SRO. As to the linking of microstructural observations with the mechanical behaviour, it is felt that the available models are somewhat precocious. The many years of efforts on $\mathrm{L}_{2}$ alloys have indeed shown that too early a model may postpone several of the key contributions. It is hoped that experimental investigations from the various groups involved in the plasticity of Al-rich TiAl alloys will continue long enough for more microstructural observations to be made available and, in particular, the possibility of an extrinsic rate-controlling mechanism be fully explored.

\section{Acknowledgements}

We gratefully thank Dr Dimiduk, Wright Patterson Air Force Base, Dayton, USA, Dr Inui and Pr Yamaguchi,
Kyoto University, Japan, for having provided us with single crystal alloys. Fruitful discussions with the above and with Dr Nakano, Osaka University, Japan are gratefully acknowledged.

\section{References}

Fu C L and Yoo M H 1990 Philos. Mag. Lett. 62159

Grégori F and Veyssière P 1999 Philos. Mag. A79 403

Hug G 1988 Weak-beam electron microscopy of dislocation splitting in TiAl relationship with plasticity, Ph.D. Thesis, Univ. Orsay, Orsay

Hug G, Loiseau A and Veyssière P 1988 Philos. Mag. A54 47

Inui H, Matsumoto M, Wu D-H and Yamaguchi M 1997 Philos. Mag. A75 395

Inui H, Chicugo K, Kishida K and Yamaguchi M 1998 Electron microscopy (eds) H A Calderon Benavides and M J Yacaman (Bristol: Institute of Physics Publishing) Vol. 2, p. 175

Jiao S, Bird N, Hirsch P B and Taylor G 1998 Philos. Mag. A78 7777

Kawabata T, Kanai T and Izumi O 1985 Acta Metall. 33 1355

Loiseau A 1985 Contribution to the study of the Ti-Al phase diagram by conventional and high resolution electron microscopy, Ph.D. Thesis, Univ. Pierre et Marie Curie, Paris

Louchet F and Viguier B 1995 Philos. Mag. A71 1313

Messerschmidt U, Bartsch M, Haussler D, Aindow M, Hattenhauer $\mathrm{R}$ and Jones I P 1995 MRS Symp. Proc. 36447

Miida R, Hashimoto S and Watanabe D 1982 Jap. J. Appl. Phys. 71 L59

Morinaga M, Saito J, Yukawa N and Adachi H D 1990 Acta Metall. 3825

Nabarro F R N and Duesbery M S 1996 Dislocations in solids (Amsterdam: Elsevier) Vol. 10

Nakano T, Matsumoto K, Seno T, Oma K and Umakoshi Y 1996 Philos. Mag. A74 251

Nakano T, Hagihira $K$, Seno T, Sumida N, Yamamoto $M$ and Umakoshi Y 1998 Philos. Mag. Lett. 78385

Nakano T, Negishi A T, Hayashi and Umakoshi Y 1999 Acta Metall. (in press)

Sriram S, Dimiduk D M, Hazzledine P M and Vasudevan V K 1997 Philos. Mag. A76 965

Stucke M A, Vasudevan V K and Dimiduk D M 1995 Mater. Sci. Engg. A192-193 111

Veyssière P 1989 MRS Symp. Proc. 133175

Viguier B, Hemker K J, Bonneville J, Louchet $\mathrm{F}$ and Martin J L 1995 Philos. Mag. A71 1295

Wiezorek J M K and Fraser H L 1998 Philos. Mag. A77 661

Woodward C and Maclaren J M 1996 Philos. Mag. A74 337

Zghal S, Menand A and Couret A 1998 Acta Mater. 46 5899 